DOI: 10.1515/awutm -2016-0009

$\overline{\mathrm{G}}$
Analele Universităţii de Vest,

Timişoara

Seria Matematică - Informatică

LIV , 1, (2016), 159- 166

\title{
Local Convergence of Exquerro-Hernandez Method
}

Ştefan Măruşter

\begin{abstract}
Local convergence of Ezquerro-Hernandez iteration is investigated in the setting of finite dimensional spaces. A procedure to estimate the local convergence radius for this iteration is proposed. Numerical experiments show that our procedure gives estimates which are very close to the maximum convergence radii.
\end{abstract}

AMS Subject Classification (2000). G.1.5

Keywords. Nonlinear equation, Iterative method, Local convergence, Ezquerro-Hernandez iteration, Local convergence radius.

\section{Introduction}

In [2] Ezquerro and Hernandes proposed a new class of third-order multipoint iterations for solving nonlinear equations $F(x)=0, F: \Omega \subset X \rightarrow Y$, where $X, Y$ are Banach spaces. The proposed class depends on the numeric parameter $t \in(0,1]$; for $t=1$ the iteration reduces to the following one point method

$$
x_{n+1}=x_{n}-F^{\prime}\left(x_{n}\right)^{-1}\left[F\left(x_{n}\right)+F\left(x_{n}-F^{\prime}\left(x_{n}\right)^{-1} F\left(x_{n}\right)\right)\right] .
$$

One important advantage of the iterations of this class consists in a better index of efficiency than Chebyshev's or Newton's classical method. Recently, 
Hernandes and Romero [3] gave the following procedure to estimate the local convergence radius for (1.1). Suppose that $p$ is a solution of the equation $F(x)=0$, there exists $F^{\prime}(p)^{-1},\left\|F^{\prime}(p)^{-1}\right\| \leq \beta$ and $F^{\prime}$ is k-Lipschitz continuous on some $B\left(p, r_{0}\right)=\left\{x:\|x-p\| \leq r_{0}\right\}$. Let $\tilde{r}=\min \left\{r_{0}, r\right\}$, where $r=\zeta_{0} /\left[\left(1+\zeta_{0}\right) \beta k\right]$ and $\zeta_{0}$ is the positive real root of the equation $t^{3}+4 t^{2}-8=0$. Then $\tilde{r}$ is a local convergence radius of (1.1).

Remark 1.1. In [3] it is noted that "this family of processes presents problems of accessibility". This remark is based on the comparison between the proposed estimation of local convergence radius for (1.1) and the estimation of local convergence radius given in [1] for Newton's method. Observe that there are compared only the estimates of the two radii and not the maximum local convergence radii, and it is not sure that the estimates keep the same ordering relation as maximum convergence radii.

In this paper we are concerned with the local convergence of the iteration (1.1). We give new convergence conditions of this iteration, presumably weaker, and propose a new procedure to estimate the local convergence radius. Our investigations are based on the convergence properties of generalized Mann iteration defined in [5]. Recall that for a given mapping $T: C \subset E \rightarrow E$ the generalized Mann iteration is defined by

$$
x_{n+1}=\left(I-D_{n}\right) x_{n}+D_{n} T\left(x_{n}\right),
$$

where $I$ is the identity mapping and $\left\{D_{n}\right\} \subset \mathcal{L}(E)$ is the "generalized control sequence" and consists of a sequence of linear mappings; usually this sequence is defined as a function of $x, D_{x}=D(x)$ (we will use the notation $D_{n}=$ $D\left(x_{n}\right)$ ), or it can be defined recursively as a mapping depending on $x_{n}$ and $D_{n-1}$. In fact (1.2) is equivalent to $x_{n+1}=x_{n}-D_{n} F\left(x_{n}\right)$ where $F(x)=$ $x-T(x)$. Iterations of this type have been extensively investigated over the years, especially the case $D_{n}=B_{n}^{-1}$; however, considering it as Mann iteration some new facts can be deduced. Recall also that a mapping $T$ : $C \subset E \rightarrow E$ with nonempty set of fixed points, Fix $(T) \neq \emptyset$, is said to be generalized demicontractive with control mapping $D: C \rightarrow \mathcal{L}(E)$ if

$$
\left\langle D_{x}(x-T(x)), x-p\right\rangle \geq \lambda\left\|D_{x}(x-T(x))\right\|^{2}, \forall x \in C, p \in F i x(T),
$$

where $\lambda>0$.

Remark 1.2. For $D_{x}=I$ the generalized demicontractivity reduces to the simple demicontractivity concept. The class of demicontractive mappings, introduced first in $[6,7]$, has several useful properties. For example, a mapping is demicontractive if and only if it satisfies the following weak quasicontractive condition

$$
\|T(x)-p\|^{2} \leq\|x-p\|^{2}+k\|x-T(x)\|^{2}, \forall x \in C, p \in F i x(T),
$$


where $k=2 \lambda-1$. Also, the class of demicontractive mappings is equivalent to the class of quasi $(L, m)$ - contractions considered in [8]. Marino and $\mathrm{Xu}$ [4] proved that the set of fixed points of a demicontractive mapping is closed and convex (they used the term "k-quasi-strict pseudo-contraction" in place of demicontractive). It can be an interesting issue whether or not these properties hold in the case of generalized demicontractive mappings, but this is not our main concern here.

\section{Preliminary lemmas}

Let $E$ denote a finite dimensional Euclidean space endowed with the standard metric and let $C$ be an open subset of $E$. Let $T: C \rightarrow E$ be a nonlinear mapping; we will assume throughout the papper that the set of fixed points of $T$ is nonempty, $\operatorname{Fix}(T) \neq \emptyset$.

Lemma 1. Suppose $T$ is Fréchet differentiable on $C$ and let $p \in C$ be a given point. Then there exists a linear mapping $R_{x, p}$ (which depends on $x, p$ ), such that

(i) $T(x)-T(p)=\left(T^{\prime}(x)+R_{x, p}\right)(x-p)$;

(ii) If $\left\|T^{\prime}(x)\right\| \leq M, \forall x \in C$ then $\left\|R_{x, p}\right\| \leq 2 M$;

(iii) For any $\varepsilon>0$, there exists $r_{\varepsilon}>0$ such that if $x \in B\left(p, r_{\varepsilon}\right)=$ $\left\{x \mid\|x-p\| \leq r_{\varepsilon}\right\}$, then $\left\|R_{x, p}\right\| \leq \varepsilon$.

The proof is straightforward if we define

$$
R_{x, p}=\frac{\left(T(x)-T(p)-T^{\prime}(x)(x-p)\right)(x-p)^{T}}{\|x-p\|^{2}} .
$$

Lemma 2. Let $F: C \rightarrow E$ be a nonlinear mapping. Suppose that $F$ is Fréchet differentiable on $C, p$ is a solution of $F(x)=0$, there exists $F^{\prime}(x)^{-1}$ and $\left\|F^{\prime}(p)^{-1}\right\| \leq \beta, \forall x \in C$. Then there exists $\tilde{r}$ such that if $x \in B(p, \tilde{r})$ then $w:=x-F^{\prime}(x)^{-1} F(x) \in B(p, \tilde{r})$.

In fact $w$ is the Newton's point and the statement of Lemma 2 is a well known result; the stronger statement $\|w-p\| \leq 0.5\|x-p\|, \forall x \in B\left(x^{*}, \tilde{r}\right)$ is also true (Theorem 5.2.1, [1]).

Lemma 3. Let $p$ be a solution of $F(x)=0$. Suppose that for all $x \in C, F$ is continuous differentiable, there exists $F^{\prime}(x)^{-1}$ and $\left\|F^{\prime}(x)^{-1}\right\| \leq \beta$. Then there exists a ball $B(p, r)$ such that the mapping $T$ defined by

$$
T(x)=x-F^{\prime}(x)^{-1}\left[F(x)+F\left(x-F^{\prime}(x)^{-1} F(x)\right)\right], x \in B(p, r),
$$


is generalized demicontractive with control function $D_{x}=F^{\prime}(x)^{-1}$ and $\lambda>$ 0.5 on $B(p, r)$.

Proof. Observe first that $p$ is a fixed point of $T$, i.e., Fix $(T) \neq \emptyset$. Let $\eta$ be a positive number such that $0<\eta<\sqrt{5}-2$. Then $(1-\eta) /(1+\eta)^{2}>0.5$ and we can choose a $\lambda$ satisfying the condition $0.5<\lambda \leq(1-\eta) /(1+\eta)^{2}$. The right hand side of this inequality is equivalent to

$$
\eta \leq \frac{-2 \lambda-1+\sqrt{8 \lambda+1}}{2 \lambda}:=s(\lambda) \text {. }
$$

Now let $M=\sup _{x \in C}\left\|F^{\prime}(x)\right\|$ and take $\varepsilon=\eta(3 \beta M+1)^{-1} \beta^{-1}$. For this value of $\varepsilon$, let $r_{\varepsilon}$ be the radius defined in Lemma 1 and let $r=\min \left\{\tilde{r}, r_{\varepsilon}\right\}$, where $\tilde{r}$ is defined in Lemma 2. From Lemma 1 we have

$$
F(x)=F(x)-F(p)=\left(F^{\prime}(x)+R_{x, p}\right)(x-p),\left\|R_{x, p}\right\| \leq \varepsilon, \forall x \in B(p, r),
$$

and from Lemma 2 it results

$$
x \in B(p, r) \Rightarrow w=x-F^{\prime}(x)^{-1} F(x) \in B(p, r) .
$$

Using again Lemma 1 we have

$$
\begin{aligned}
F(w) & =F(w)-F(p)=\left(F^{\prime}(w)+R_{w, p}\right)\left(x-p-F^{\prime}(x)^{-1} F(x)\right) \\
& =\left(F^{\prime}(w)+R_{w, p}\right)\left[I-F^{\prime}(x)^{-1}\left(F^{\prime}(x)+R_{x, p}\right)\right](x-p) \\
& =-\left(F^{\prime}(w)+R_{w, p}\right) F^{\prime}(x)^{-1} R_{x, p}(x-p) .
\end{aligned}
$$

where $\left\|R_{w, p}(x)\right\| \leq 2 M$. Therefore

$$
F(x)+F(w)=\left[F^{\prime}(x)+R_{x, p}-\left(F^{\prime}(w)+R_{w, p}\right) F^{\prime}(x)^{-1} R_{x, p}\right](x-p),
$$

and

$$
\begin{aligned}
& F^{\prime}(x)^{-1}(x-T(x)) \\
& =\left[I+F^{\prime}(x)^{-1} R_{x, p}-F^{\prime}(x)^{-1}\left(F^{\prime}(w)+R_{w, p}\right) F^{\prime}(x)^{-1} R_{x, p}\right](x-p) .
\end{aligned}
$$

Using the notation $\Delta_{x}=\left[-F^{\prime}(x)^{-1}+F^{\prime}(x)^{-1}\left(F^{\prime}(w)+R_{w, p}\right) F^{\prime}(x)^{-1}\right] R_{x, p}$ we have

$$
F^{\prime}(x)^{-1}(x-T(x))=\left(I-\Delta_{x}\right)(x-p), \forall x \in B(p, r) .
$$

For $\Delta_{x}$ we have that

$$
\left\|\Delta_{x}\right\| \leq(3 \beta M+1) \beta\left\|\bar{R}_{x, p}\right\| \leq \eta \leq s(\lambda) .
$$

The rest of the proof follows verbatim the proof of Lemma 3 [5]. 


\section{Local convergence}

Our approach is based on the following theorem concerning the convergence of the generalized Mann iteration [5]:

Theorem 3.1. Let $p$ be a fixed point of $T$ and $r_{0}>0$ such that $B\left(p, r_{0}\right) \subset C$. Suppose $T$ and $D$ satisfy the following conditions:

(i) $I-T$ is demiclosd at zero on $B\left(p, r_{0}\right)$;

(ii) $D_{x}$ is invertible and $\left\|D_{x}^{-1}\right\| \leq M, \forall x \in B\left(p, r_{0}\right)$;

(iiI) $T$ is generalized demicontractive on $B\left(p, r_{0}\right)$ with control function $D$ and $\lambda>0.5$.

Then the sequence given by (1.1) converges to a fixed point of $T$ for any starting point in $B\left(p, r_{0}\right)$.

Note that this theorem requires only a weak smoothness property of $\mathrm{T}$ (demiclosedness of $I-T$ at zero) and that condition (ii) is satisfied for a large class of linear mappings. A typical case of mapping $T$ which is not continuous but satisfies the demiclosedess condition is the cyclic projection mapping appearing in the projection type algorithms for solving convex feasibility problems.

Theorem 3.2. Suppose that $F$ is continuous differentiable, there exists $F^{\prime}(x)^{-1}$ and $\left\|F^{\prime}(x)^{-1}\right\| \leq \beta$ for all $x \in C$. Then Fix $(T)$ is made of isolated points and the sequence $\left\{x_{n}\right\}$ given by (1.1) converges locally to some fixed point.

Proof. Let $p$ be a fixed point of $T$ and let $c$ be such that $0<c<\beta^{-1}$. Take $\varepsilon=c$ in Lemma 1 and let $r_{c}$ be the radius defined in the same Lemma. We have

$$
\|F(x)\|=\left\|\left(F^{\prime}(x)+R_{x, p}\right)(x-p)\right\| \geq\left\|\left(F^{\prime}(x)+R_{x, p}\right)^{-1}\right\|^{-1}\|x-p\|,
$$

and $\left\|R_{x, p}\right\| \leq c$ for all $x \in B\left(p, r_{c}\right)$. From Banach lemma we obtain $\|\left(F^{\prime}(x)+\right.$ $\left.R_{x, p}\right)^{-1} \| \leq \beta /(1-c \beta)$. Therefore

$$
\|x-p\| \leq \frac{\beta}{1-c \beta}\|F(x)\|, \forall x \in B\left(p, r_{c}\right) .
$$

This shows that $p$ is the unique fixed point in $B\left(p, r_{c}\right)$. Now let $r_{0}=$ $\min \left\{r_{c}, r\right\}$, where $r$ is the radius defined in Lemma 3. The fulfillment of conditions (i)-(iii) of Theorem 3.1 on $B\left(p, r_{0}\right)$ is now very easy to check. Indeed, the conditions (i) and (ii) are obviously satisfied and (iii) results from Lemma 3. We can apply Theorem 3.1 to deduce the convergence of the sequence $\left\{x_{n}\right\}$ generated by (1.1) for any starting point in $B\left(p, r_{0}\right)$. 
The local convergence of the general Ezquerro-Hernanadez iteration is proved in [3] (Theorem 1) under the following two condition: $(\mathrm{C} 1)$ there exists $F^{\prime}(p)^{-1}$ and $\left\|F^{\prime}(p)^{-1}\right\| \leq \beta,(\mathrm{C} 2) F^{\prime}$ is $K$ Lipschitz continuous oc $C$. It is simple to show that from these two conditions it follows that there exists $F^{\prime}(x)^{-1}$ and $\left\|F^{\prime}(x)^{-1}\right\| \leq \beta$ on some ball in $C$.

Based on Theorem 3.1 we can construct an algorithm that estimates quite well the local convergence radius for (1.1). Supposing that conditions (i) and (ii) of Theorem 3.1 are fulfilled, the idea of this algorithm is to find the largest value for $r$ such that the mapping $T$ satisfies condition (iii).

The main steps of the algorithm are:

1. Apply a search line algorithm (for example of the type half-step algorithm) on the positive real axis to find the largest value for $r$;

2. At every step of 1 solve the following constraint optimization problem

$$
m=\min _{x \in B(p, r)} \frac{\left\langle D_{x}(x-T(x)), x-p\right\rangle}{\left\|D_{x}(x-T(x))\right\|^{2}},
$$

and verify condition $m>0.5$.

Note that the minimum in step 2 must be global on $B(p, r)$ so that the effective computation of this value is not an easy task. In our numerical experiments we use several optimization algorithms available in any common mathematical software.

\section{Numerical experiments}

This section is devoted to numerical experiments in order to evaluate the efficiency of the proposed procedure. More precisely, we investigate how close are the estimates computed with the proposed algorithm to the maximum local convergence radii. To this end we need the attraction basin of considered fixed point and then we can estimate the maximum convergence radius. We check the convergence of iteration process starting from all points of a given net of points and select those points for which the iteration (1.1) converges. Of course, the basin computed in this numerical way, generally have informative character. However, it gives significant information on the attraction basin and the efficiency of the proposed algorithm can be satisfactorily evaluated.

Applying this numerical procedure, we performed a large number of numerical experiments in one or several dimensions; below is presented a small 
part of them.

In the first experiment we have computed the local convergence radius with the proposed algorithm and the maximum radius for a significant number of real functions.. In all these experiments the two radii coincide. For example, in the case of the function $f(x)=0.5 x^{2}+\cos (x)$ with $p=1.04855836 \ldots$ the estimate is $r=0.58308619 \ldots$ and the maximum radius is $r_{m}=0.58308619 \ldots$

In the second experiment we applied our algorithm to several mappings in two variables. For the following three of them:

$$
\begin{gathered}
\text { (a) } F(x)=\left(\begin{array}{l}
0.8 x_{1}-\cos \left(x_{1}\right)+x_{2}^{2}+1 \\
x_{1}^{3}+0.8 x_{2}
\end{array}\right),(b) F(x)=\left(\begin{array}{c}
3 x_{1}^{2}-x_{1} x_{2}^{3}+3 x_{2} \\
2 x_{1}+x_{2}^{3}-0.2 x_{2}
\end{array}\right), \\
\left(\text { c) } F(x)=\left(\begin{array}{l}
x_{1} x_{2}^{3}-x_{1}+2 x_{2}^{2} \\
x_{1}^{2}+\sin \left(x_{2}\right)
\end{array}\right),\right.
\end{gathered}
$$

the results are given in Figure 1.

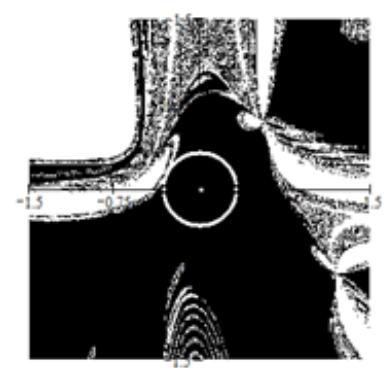

(a)

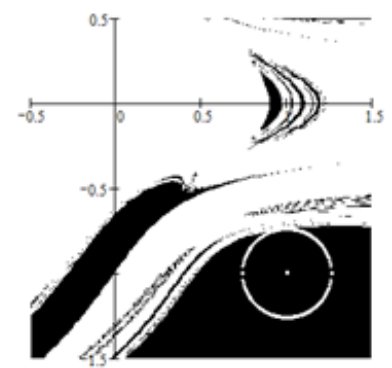

(b)

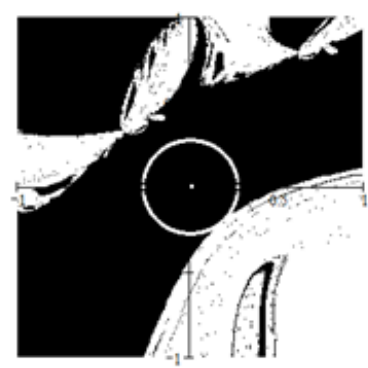

(c)

Figure 1: The estimate of local convergence radii with proposed algorithm

The black areas represent the whole attraction basins and the white circles the local convergence balls. It can be seen that the proposed algorithm gives convergence radii very close to the maximum ones or even it gives the maximum convergence radii.

Remark 4.1. The following problem is open: How large is the class of mappings for which the proposed algorithm gives local convergence radius close to maximum radius? Our experiences show that for a relatively large class of mappings the proposed procedure works. It is worthwhile to underline that this favorable situation holds for Ezquerro-Hernandez method. We applied the same procedure to other five (classical) methods, Newton, Simplified Newton, Cebyshev, Successive Approximations and Gradient: the 
results were relatively negative, the closeness of estimates to the maximum convergence radii being lost.

\section{References}

[1] J.E. Dennis and R.B. Schnabel, Numerical Methods for Unconstrained Optimization and Nonlinear Equation, SIAM: Philadelphia, PA, USA, 1996

[2] J.A. Ezquerro and M.A. Hernandez, An optimization of Chebyshev's method, Journal of Complexity, 25, (2009), 343-361

[3] M. A. Hernandez-Veron and N. Romero, On the Local Convergence of a Third Order Family of Iterative Processes, Algorithms, 8, (2015), 1121-1128

[4] G.Marino and H-K.Xu, Weak and strong convergence theorems for strict pseudocontractions in Hilbert spaces, J. Math. Anal. Appl., 329, (2007), 336-346

[5] St. Maruster, Local convergence radius for the Mann-type iteration, Analele Universitatii de Vest, Timisoara Seria Matematica si Informatica, LIII, 2, (2015), 109-120

[6] St.Maruster, The solution by iteration of nonlinear equations in Hilbert spaces, Proc. Amer. Math.Soc., 63 (1), (1977), 69-73

[7] St. Maruster, Sur le calcul des zeros d'un operateur discontinu par iteration, Canad. Math. Bull., 16 (4), (1973), 541-544

[8] Y.Qin and B.E.Rhoades, T-stability of Picard iteration in metric spaces, Fixed Point Theory and Applications, Hindawi Publ. Corp., 2008 (Article ID418971)

Ştefan Măruşter

Department of Computer Science

West University of Timisoara

B-l V. Parvan nr. 4

Timisoara

Romania

E-mail: maruster@info.uvt.ro

Received: 18.04.2016

Accepted: 5.05.2016 